Birgit Sandkaulen*

\title{
Der Begriff des Lebens in der Klassischen Deutschen Philosophie - eine naturphilosophische oder lebensweltliche Frage?
}

https://doi.org/10.1515/dzph-2019-0068

\begin{abstract}
In this paper, I point to two connotations that come with the concept of 'life': it may refer to the natural phenomenon of organic life studied by the "life sciences" and philosophy of nature - Naturphilosophie - but it may equally refer to the lives we lead in a complex lifeworld. Of course, natural features belong to the lifeworld as well. However, the lifeworld is also shaped by various individual and cultural practices and, as such, it is not reducible to the natural. I defend a twofold claim: first, a genuine interest in the lifeworld informs Classical German Philosophy throughout on a meta-level; second, it is this interest in the lifeworld that grounds and motivates considerations of natural phenomena in Classical German Philosophy. I trace the shared interest in the lifeworld from Hegel, Schelling, and Fichte back to Friedrich Heinrich Jacobi's formative ideas. Jacobi profoundly influenced the debates in Classical German Philosophy with his "resolute realism."
\end{abstract}

Keywords: life, lifeworld, Classical German Philosophy, German Idealism, Hegel, Schelling, Fichte, Jacobi

\section{Zwei Bedeutungen des Begriffs „Leben“}

Woran denkt man spontan beim Ausdruck „Leben“? Eine der allerersten Assoziationen ist ganz sicher die, an die Natur, an etwas Natürliches zu denken - und zwar mit dem spezifizierenden Zusatz der organischen Natur. Hier stößt man dann auch gleich auf den Begriff des „Lebewesens“. Lebewesen, so wird der Begriff bei Wikipedia definiert, sind „organisierte Einheiten, die unter anderem zu Stoff-

*Kontakt: Birgit Sandkaulen, Institut für Philosophie I, Ruhr-Universität Bochum, 44780 Bochum; birgit.sandkaulen@rub.de 
wechsel, Fortpflanzung, Reizbarkeit, Wachstum und Evolution fähig sind““. ${ }^{1}$ Dazu zählen alle Organismen. Auch Menschen sind in diesem Sinne Lebewesen oder „animalia“, wie es die alte Definition des Menschen als „animal rationale“ ausgesagt und dabei der Grundbestimmung des Lebewesens zugleich eine charakteristische Sonderbestimmung - die Rationalität - beigefügt hat.

$\mathrm{Zu}$ dieser auf die organische Natur verweisenden Semantik des Lebensbegriffs passt, dass sich in den letzten Jahren eine Gruppe von Wissenschaften zu den sog. „life sciences“, den „Lebens“- oder „Biowissenschaften“ zusammengeschlossen hat. $\mathrm{Zu}$ diesen Wissenschaften zählen neben Biologie auch Medizin, Biomedizin, Biochemie, Molekularbiologie, Biophysik, Bioinformatik und Humanbiologie. Je nach Ausrichtung liegt der Akzent dieser Wissenschaften entweder auf Interessen der Grundlagenforschung oder der angewandten Forschung, und jeder ahnt oder weiß, welch riesiges - im übrigen auch ökonomisch interessantes - Forschungsfeld sich hier eröffnet hat.

Spätestens an dieser Stelle ist darum auch zu ergänzen, dass das Thema „Leben“ im Augenblick Konjunktur hat. Die „life sciences“ ziehen die forschungspolitische Aufmerksamkeit auf sich - und im Gefolge dieser Aufmerksamkeit, dies ist jedenfalls meine These, ist die Frage nach der lebendigen Natur auch philosophisch brisant geworden. Auf die unzähligen „Naturalismus“-Debatten will ich hier gar nicht eingehen. Ich möchte lediglich hervorheben, dass wir es nach meinem Eindruck derzeit mit so etwas wie einem Strategiewechsel zu tun haben. Anstatt gegenüber allen möglichen Naturalisierungstendenzen die Domäne der Philosophie mit dem Argument zu verteidigen, dass „Geist“ oder „Kultur“ oder auch - in Erinnerung an die vorhin erwähnte Definition des Menschen als „animal rationale“ - „Vernunft“ und „Rationalität“ etwas anderes sind als Natur, wendet man sich philosophisch dem Begriff des Lebens als einem Grundbegriff selber zu.

Vor diesem Hintergrund möchte ich die biologische Semantik des Lebensbegriffs als eine im weitesten Sinne naturphilosophische Bestimmung bezeichnen. Im weitesten Sinne deshalb, weil sie die menschliche Natur - das menschliche Lebewesen - in sich einschließt und auch offen dafür ist, spezifische Eigenschaften des menschlichen Lebewesens als besondere Ausprägung seiner Natur zu verstehen, anstatt sie reduktionistisch auf so etwas wie die „eigentliche“ Natur zurückzuführen.

Jedoch gibt es mindestens noch eine weitere Konnotation des Begriffs „Leben“. Mit einem Ausdruck, den ich mir von der phänomenologischen Tradition ausleihe, bezeichne ich diese Konnotation als lebensweltliche Bestim-

1 https://de.wikipedia.org/wiki/Lebewesen (26.2.2019). 
mung des Begriffs Leben. Ganz unabhängig davon, was die Phänomenologie im Anschluss an den späten Husserl darunter versteht (dem werde ich im Folgenden nicht nachgehen), halte ich das Wort „Lebenswelt“ für ein ganz wunderbares, außerordentlich glückliches Wort. Was ist damit gemeint?

Wie der Begriff wörtlich sagt, liegt der Fokus hier nicht auf der Natur, sondern auf der Welt. Gemeint ist die Welt, in der wir leben, was selbstverständlich die natürliche Welt nicht ausschließt, sondern integriert. Gemeint ist zugleich, dass wir „weltoffene“ Wesen sind, wir vollziehen unser Leben als ein „In-der-WeltSein“. Und dementsprechend gehören zur „Lebenswelt“ auch die persönlichen und intersubjektiv geteilten Erfahrungen, Einstellungen und Überzeugungen hinzu, die unser Leben strukturieren. In diesem Sinn wird mit dem Ausdruck „Lebenswelt“ der kosmologische Akzent der „Welt“ als eines in sich strukturierten Ganzen auf die Wirklichkeit hin fokussiert, die sich uns in unseren Lebensvollzügen $\mathrm{zu}$ verstehen gibt und die wir zugleich gestalten. Nicht zu vergessen ist bei alledem der kritische Akzent des Begriffs „Lebenswelt“, der die Prägung dieses Begriffs wesentlich mitgetragen hat. Diese kritische Pointe ergibt sich aus dem Kontrast der „Lebenswelt“ zur wissenschaftlich-theoretischen Einstellung zur Welt. Die kritische ,vorwissenschaftliche“ Akzentuierung rückt den Begriff in die Nähe des Common Sense, womit dann nicht der sogenannte „gesunde Menschenverstand“ gemeint ist, sondern das Insgesamt unseres In-der-Welt-Seins mit den zugehörigen Überzeugungen und Einstellungen, die unser Leben ausmachen. Diesen kritischen Sinn des Ausdrucks „Lebenswelt“ hat Jürgen Habermas mit der „Teilnehmerperspektive“ bezeichnet, die von der „Beobachterperspektive“ der Wissenschaft zu unterscheiden ist. ${ }^{2}$ Dies erscheint mir hilfreich, um den „lebensweltlichen“ Fokus richtig zu justieren.

2 Habermas (1981), 179. Auf die hier einschlägige Abgrenzung eines kommunikativen Begriffs der Lebenswelt gegenüber dem bewusstseinsphilosophischen Paradigma Husserls gehe ich an dieser Stelle ebenfalls nicht näher ein, wenngleich ich selbst im Folgenden mit Jacobi den „Idealismus“ der Bewusstseinsphilosophie auch meinerseits kritisiere. Der heute allgemein auf Wittgenstein zurückgeführte, tatsächlich aber bis auf Schleiermacher zurückreichende Begriff der „Lebensform“ teilt mit der „Lebenswelt“ die nicht-biologische Semantik, scheint mit der Akzentuierung der „Form“ jedoch eine eingeschränktere Bedeutung zu haben, der gegenüber der weite mundane Charakter der „Lebenswelt“ für die folgenden Überlegungen vorzuziehen ist. 


\section{Die lebensweltliche Bedeutung des Lebens als Metabegriff der klassischen deutschen Philosophie}

Was haben diese einleitenden Überlegungen zur Semantik des Lebensbegriffs mit der klassischen deutschen Philosophie zu tun? Dem Sachregister des Handbuchs Deutscher Idealismus zufolge müsste die Antwort lauten: nichts. ${ }^{3}$ Nur „Lebenskraft“ wird einmal mit Verweis auf Johann Friedrich Blumenbach genannt. Die Sache ist kurios, weil der Artikel „Natur“ in diesem Handbuch den Begriff „Leben“ oftmals erwähnt. Das heißt: Aus der Leerstelle im Handbuch-Register den Schluss zu ziehen, der Begriff des Lebens spiele im sogenannten „Deutschen Idealismus“ keine Rolle, wäre ganz falsch. Wer immer das Sachregister angefertigt hat, war für das Thema seinerzeit offenkundig noch nicht empfindlich, was heutzutage ganz undenkbar wäre. Indessen muss man noch einen Schritt weitergehen.

Dass das „Leben“ im Sachregister fehlt, zeigt nicht nur die fehlende Sensibilität für den naturphilosophischen Lebensbegriff an, der im Artikel selbst de facto vorkommt, sondern auch die fehlende Sensibilität für den von mir eben skizzierten lebensweltlichen Begriff des Lebens. Dieser Begriff lässt sich in den Sparten des Handbuchs - „Die Vernunft und das Absolute“, „System und Methode“, „Erkenntnis und Wissen“, „Natur“, „Freiheit, Moral und Sittlichkeit“, „Recht und Staat“, „Geschichte“, „Religion und Gottesbegriff“ sowie schließlich „Das Schöne und die Kunst“ - allerdings auch gar nicht abbilden und rubrizieren. Der lebensweltliche Begriff des Lebens verweist nicht auf ein spezielles Gebiet, vielmehr liegt er der Auseinandersetzung mit solchen speziellen Gebieten insgesamt zugrunde.

Damit habe ich nun bereits zwei Thesen aufgestellt. Den wörtlichen Ausdruck „Lebenswelt“ gibt es in der klassischen deutschen Philosophie nicht, aber der Sache nach, so behaupte ich erstens, ist diese Bedeutung des Lebens in der Epoche ganz präsent. Wir haben es hier also nicht nur mit der naturphilosophischen Signatur des Begriffs zu tun. Eine zweite, noch weitergehendere These schließe ich daran an. Ich möchte zeigen, dass das Leben im lebensweltlichen Sinn als so etwas wie ein Metabegriff zu verstehen ist. Das Interesse an der Lebenswelt grundiert alle Bemühungen der Epoche. Insofern tritt dieses Interesse nicht gleichsam neben das naturphilosophische Interesse als Ergänzung eines weiteren Aspekts. Vielmehr geht das Interesse an der Lebenswelt dem naturphiloso-

3 Vgl. Sandkühler (2005). 
phischen Lebensbegriff voraus und bestimmt damit auch die Konturen dessen, was naturphilosophisch zur Verhandlung steht. Dieser Zusammenhang scheint mir auffallend und von großer, auch aktueller Virulenz zu sein. An dieser Stelle kann ich ihn natürlich nur skizzenhaft umreißen.

\section{Textbelege im Ausgang von Jacobi}

Um meine doppelte These nicht im luftleeren Raum zu platzieren, beginne ich bewusst mit einer Reihe von etwas ausführlicheren Textbelegen, die zunächst einmal einen Eindruck von der Sache vermitteln sollen.

1. Jacobi im David Hume (1787): Die „substanzielle Form der organischen Wesen [...] ist dasjenige, was ich im eigentlichsten Verstande mich selbst nenne, und von dessen Realität ich die vollkommenste Ueberzeugung, das innigste Bewustseyn habe, weil es die Quelle selbst meines Bewustseyns, und das Subject aller seiner Veränderungen ist. Die Seele, um eine Vorstellung von sich selbst zu haben, müßte sich von sich selbst unterscheiden, sich selbst äusserlich werden können. Von dem, was Leben ist, haben wir gewiß das innigste Bewustseyn; aber wer kann sich vom Leben eine Vorstellung machen? [...] Und nichts anders ist unsere Seele, als eine bestimmte Form des Lebens. Ich weiß nichts verkehrteres, als das Leben zu einer Beschaffenheit der Dinge zu machen, da im Gegentheil die Dinge nur Beschaffenheiten des Lebens, nur verschiedene Ausdrücke desselben sind; denn das Mannichfaltige kann im Lebendigen allein sich durchdringen und Eins werden. Wo aber Einheit, reale Individualität aufhört, da hört alles Daseyn auf “. ${ }^{4}$

2. Schelling in der Ichschrift (1795): Auf die Frage, „wohin eigentlich solche Grundsäze, die man als ganz neue aufstellt, führen sollen, ob sie ein bloßes Eigenthum der Schule bleiben sollen, oder in's Leben selbst übergehen werden [so ist zu bemerken], daß eine Philosophie, die auf das Wesen des Menschen selbst gegründet ist, nicht auf todte Formeln, als eben so viele Gefängnisse des menschlichen Geistes, oder nur auf ein philosophisches Kunststück gehen könne, das die vorhandenen Begriffe nur wieder auf höhere zurükführt und das lebendige Werk des menschlichen Geistes in todte Vermögen begräbt; daß sie vielmehr, wenn ich es mit einem Ausdruk Jacobi's sagen soll, darauf geht, Daseyn zu enthüllen und zu offenbaren, daß also ihr Wesen, Geist, nicht Formel und Buchstabe, ihr höchster Gegenstand aber nicht das durch Begriffe vermittelte, mühsam in Begriffe zusammengefaßte, sondern das unmittelbare nur sich selbst gegenwärtige im Menschen seyn müsse“. 5

3. a) Hegel im Fragment Absolute Entgegensezung (1800): „Diese Erhebung des Menschen nicht vom Endlichen zum Unendlichen, denn dieses sind nur Produkte der blossen Refle-

4 Jacobi (2004), 83-84 (alle Hervorh. in 1. bis 4. im Orig.).

5 Schelling (1980), 76-77. 
xion, und als solcher ist ihre Trennung absolut - sondern vom endlichen Leben zum unendlichen Leben - ist Religion.“6

b) Hegel in der Differenzschrift (1801): „Wenn die Macht der Vereinigung aus dem Leben der Menschen verschwindet und die Gegensätze ihre lebendige Beziehung und Wechselwirkung verloren haben und Selbständigkeit gewinnen, entsteht das Bedürfnis der Philosophie.“7

4. Fichte im Sonnenklaren Bericht (1801): „Ich erkläre sonach hiermit öffentlich, dass es der innerste Geist und die Seele meiner Philosophie sey: der Mensch hat überhaupt Nichts, denn die Erfahrung, und er kommt zu allem, wozu er kommt, nur durch die Erfahrung, durch das Leben selbst. Alles sein Denken, sey es ungebunden oder wissenschaftlich, gemein oder transcendental, geht von der Erfahrung aus, und beabsichtigt hinwiederum Erfahrung. Nichts hat unbedingten Werth und Bedeutung, als das Leben; alles übrige Denken, Dichten, Wissen hat nur Werth, insofern es auf irgendeine Weise sich auf das Lebendige bezieht, von ihm ausgeht, und in dasselbe zurückzulaufen beabsichtigt.“8

So weit einige exemplarische Textbelege, denen sich viele weitere beifügen ließen. Für den vorliegenden Zweck sollten sie aber hinreichend sein. Bevor ich auf die inhaltlichen Punkte näher eingehe, schicke ich einige kleine Anmerkungen voraus.

1. In der Reihe der Texte fehlt Kant - das ist kein Zufall. Was hier im Namen des "Lebens“ besprochen wird, fasst Kant unter dem Namen des „Weltbegriffs“ der Philosophie. „Welt“ kommt bei Kant also vor, aber dass das Leben in diesem Zusammenhang fehlt, ist signifikant und muss bei anderer Gelegenheit vertieft werden.

2. Ein Zufall ist es gleichfalls nicht, dass Jacobi die Reihe eröffnet und dass die Texte von Schelling, Hegel und Fichte alle in einem intimen Bezug zu Jacobi stehen. Über Jacobis oben zitierte Schrift David Hume über den Glauben oder Idealismus und Realismus hinaus ist hier natürlich insbesondere auch sein Buch Über die Lehre des Spinoza in Briefen an den Herrn Moses Mendelssohn (1785/1789) relevant. Dass die postkantische Philosophie ohne Jacobi überhaupt nicht verständlich wird, ist inzwischen anerkannter - vielleicht aber noch nicht überall auch realisierter - Stand der Forschung. Jedoch möchte ich hier keine „Einflussforschung" betreiben. Es geht darum, dass Jacobi ein Motiv stark gemacht hat, dem sich die jüngere Generation in gar keiner Weise entziehen konnte oder gar wollte. Mit Hegels Worten anlässlich von Jacobis Tod gesagt: „Er war einer von denen, die einen Wendepunkt der geistigen Bildung der Zeit sowie der Individuen

6 Hegel (2014), 343.

7 Ders. (1968), 14.

8 Fichte (1971), 333-334. 
formierten und die für die Welt, in der wir uns unsere Existenz vorstellen, einer der festen Halte waren“ (Brief an Niethammer, 26.3.1819). Da ist der Ausdruck „Lebenswelt“ ja fast schon von Hegel formuliert.

3. Die Textauszüge reichen bis 1801, sie stammen also aus der Formationszeit der nachkantischen Philosophie. Der Blick auf die Anfänge ist aus systematischen Gründen wichtig, weil man gerade hier die Herausbildung einer spezifischen Interessenlage sieht.

\section{Wirklichkeit}

Worum geht es in den zitierten Texten? Dass das Leben hier jeweils eine zentrale Rolle spielt, ist evident. Auch zeichnet sich ab, dass es Unterschiede zwischen den Positionen gibt, wovon später die Rede sein soll. In einem ersten Schritt soll es zunächst um einige gemeinsame Merkmale gehen, und hier fällt zuallererst auf, dass es sich um eine naturphilosophische Signatur des Lebensbegriffs offenkundig nicht handelt. Schelling hat um diese Zeit (1795) seine Naturphilosophie noch nicht einmal im Ansatz entwickelt. Fichte liegt der naturphilosophische Ansatz überhaupt ganz fern. Bezeichnenderweise wird er aber wenig später, 1804, seine Wissenschaftslehre im Ganzen in eine „Philosophie des Lebens“ umformatieren, was mit der Vorlesung von 1806 Anweisung zum seligen Leben bekräftigt wird. Hegel schließlich vermerkt in dem genannten Fragment von 1800 sogar ausdrücklich, dass die „Natur nicht selbst Leben, sondern ein von der Reflexion obzwar aufs würdigste behandeltes, fixirtes Leben“ sei, und eben diese Fixierung in der Erhebung vom endlichen zum unendlichen Leben aufgelöst werde. ${ }^{9}$ Dass man in der Reihe der Texte das naturphilosophische Moment in der Adresse an das „organische Wesen“ noch am ehesten bei Jacobi findet, ist durchaus interessant. Tatsächlich hat Jacobi im David Hume vor Kants Kritik der Urteilskraft eine biologische Sicht auf die Natur eröffnet, auf deren Grundzüge sich Schelling später in seinen naturphilosophischen Entwürfen auch erkennbar stützen wird.

Gleichwohl ist es Jacobi nicht um eine naturphilosophische Argumentation zu tun. Umgekehrt ist sein Text das erste Belegstück für meine These, dass ausgehend vom lebensweltlichen Begriff des Lebens als einem „Metabegriff“ auch eine neue Verständigung über die Natur in Gang kommen kann. Beachtet man den Kontext, dann wird die Stoßrichtung Jacobis ganz klar: Bevor man bestimmte Phänomene der Welt genauer auszeichnen und zu anderen Phänomenen ins Ver-

9 Hegel (2014), 342. 
hältnis setzen kann, muss es allem voran darum gehen, die Wirklichkeit dieser Phänomene überhaupt zur Geltung zu bringen. Das ist das fundamentale Motiv, das Jacobi ausdrücklich unter den Titel eines „entschiedenen Realismus“ rückt. Und wie das im David Hume geführte Gespräch eindringlich zeigt, ist die Position dieses „entschiedenen Realismus“ gerade keine erkenntnistheoretische Position. Als erkenntnistheoretische Verirrungen der Moderne werden vielmehr eine ganze Reihe von Positionen (Rationalismus, Empirismus, Skeptizismus, transzendentaler Idealismus) kritisiert, mit dem Argument nämlich, dass sie sich als Varianten des „Idealismus“ sämtlich in die isolierte Stellung des Bewusstseins zurückgezogen haben, um von der Welt folglich nur noch im aporetischen Modus der Vorstellung zu sprechen. Kants Behauptung, dass wir es nur mit Erscheinungen, aber nicht mit den Dingen an sich zu tun haben, ist in Jacobis Augen das äußerste Skandalon auf diesem Weg der neuzeitlichen Philosophie, demgegenüber es in entschieden realistischer Weise unsere basale Überzeugung der Wirklichkeit zu verteidigen gilt. Worauf stützt sich aber diese Überzeugung, wenn nicht auf den Erfahrungszusammenhang unseres Lebens?

Man sieht: Bereits in diesem basalen Sinne, noch vor seiner spezifischen Bedeutung und Ausprägung also, ist der Metabegriff „Leben“ ein kritischer Begriff. Diesen Begriff in die Auseinandersetzung einzuführen und sich auf das Leben als relevante Größe zu beziehen, heißt, sich allen möglichen Theoriekonstrukten zu widersetzen, die die Wirklichkeit verstellen, wobei es zum kritischen Format des Lebensbegriffs gehört, wie gerade Jacobis Gespräch demonstriert, den Bezug auf das Leben nicht naiv zu beschwören, sondern sich dieser Dimension allererst im Durchgang durch eine gründliche Analyse der Bewusstseinsphilosophie zu vergewissern. ${ }^{10}$

Indessen lässt sich dieser Zusammenhang, wonach die Rede vom Leben gegenüber den Abstraktionen von Theoriekonstrukten das substantielle Interesse an Wirklichkeit vertritt, nicht nur bei Jacobi erkennen. Motiviert durch Jacobi wird dieser Zusammenhang zu einem gemeinsamen Merkmal der nachkantischen Philosophie. Eine Philosophie, die sich nicht für Wirklichkeit interessiert und eben deshalb aus dem Leben und für das Leben spricht, scheidet als Option vollständig aus. Diese Überzeugung belegen alle von mir zitierten Textauszüge: Philosophie, so Schelling, soll keine „toten Formeln“ produzieren und ein „philosophisches Kunststück“ aufführen, sondern ,ins Leben selbst übergehen“; Philosophie, so Hegel, entsteht mit dem „Bedürfnis“, das Leben über sich selbst zu

10 Vgl. hierzu ausführlich Sandkaulen (2019), 135-167. 
verständigen $^{11}$; und Fichte zufolge hat nichts ,unbedingten Wert und Bedeutung als das Leben“, aus dessen Erfahrungszusammenhang alles andere seine Relevanz und Geltung bezieht.

Als Grundüberzeugung wandert dieser Komplex sozusagen in die Fundamente der nachkantischen Entwürfe ein und verbindet die Positionen sogar genau in dem Maße, wie sie sich alsbald heillos zerstreiten: Der stehende Vorwurf in diesem Streit ist ja eben der, dem Wirklichkeitsanspruch entgegen doch nur „formalistisch“ geblieben zu sein. In diesem Sinne und ungeachtet aller internen Debatten um die Konzepte von „Idealismus“ und „Realismus“ ist die nachkantische Philosophie im Kern realistisch grundiert. Auch und nicht zuletzt deshalb führt der Name „Deutscher Idealismus“, der der Epoche maßgeblich von dem Neukantianer Windelband aufgeprägt worden ist, vollständig in die Irre und zu zahllosen Missverständnissen ihrer Grundanliegen. ${ }^{12}$ Und umgekehrt ist es alles andere als verwunderlich, dass das „realistische“ Potential der klassischen deutschen Philosophie gerade gegenwärtig ins Zentrum rückt, in einer Zeit, die ihrerseits sichtlich „realismusorientiert“ ist.

\section{Der kritische Begriff der Lebenswelt: Inhaltliche und epistemische Grundzüge}

Ich hoffe, dass ich mit dem bisher Gesagten verdeutlichen konnte, welchen Einstieg man nehmen muss, um auf die fundamentale Relevanz des Lebensbegriffs zu stoßen. Aber, so möchte man vielleicht längst fragen: Was genau ist denn nun das Leben? Was füllt diesen Begriff, wenn er nicht einfach nur ein anderes Wort für „Wirklichkeit“ ist? Wenn vielmehr gelten soll, dass die Wirklichkeit in genau dem Maße wirklich wirklich, also im emphatischen Sinne wirklich - und keine kantische Erscheinung oder eine repräsentationalistische Vorstellung - ist, wie sie sich als lebendig zeigt? Auf diese Überzeugung läuft die Sache ja hinaus. Daher ist die genannte Frage selbstverständlich wichtig. Ich ignoriere sie keineswegs und werde versuchen, sie wenigstens in Umrissen $\mathrm{zu}$ beantworten. Zunächst

11 Besonders eindrucksvoll hat Hegel dieses Anliegen dann auch in seinen ersten Jenaer Vorlesungen 1801/02 formuliert: „Was das allgemeine des Bedürfnisses der Philosophie betrifft, so wollen wir es in der Form einer Antwort auf die Frage, welche Beziehung hat die Philosophie aufs Leben? klar zu machen suchen, eine Frage, die eins ist mit der: inwiefern ist die Philosophie praktisch? Denn das wahre Bedürfniß der Philosophie geht doch wohl auf nichts anders als darauf, von ihr und durch sie leben zu lernen“ (Hegel 1998, 261).

12 Vgl. hierzu ausführlich Jaeschke (2000) u. Sandkaulen (2007). 
jedoch ist ein anderer Punkt zu betonen. Es ist kein äußerlicher Aspekt, sondern es gehört zur Sache selbst hinzu, dass man eigentlich im Blick auf das Leben eine solche „Was-Frage“ nicht stellen und auf eine Was-Frage eigentlich auch keine Antwort erwarten kann. Zugespitzt formuliert bedeutet das: Der Einsatz des Lebensbegriffs steht genau für das, was sich allen Was-Fragen entzieht. Leben ist kein Objekt.

Ich möchte diesen Punkt den mit dem Inhalt des Lebensbegriffs untrennbar verbundenen epistemischen Aspekt nennen, der eigentlich auch zur Folge hat, dass es sich um einen „Begriff“ im üblichen Sinn gar nicht handelt. Auch insofern ist der Lebensbegriff ein kritischer Begriff: Die Rationalitätskritik, die in seinem Gebrauch, also performativ, zum Ausdruck kommt, ist Moment seiner inhaltlichen Bestimmung, und dies lässt sich ebenfalls als ein gemeinsames Merkmal in allen Textbelegen diagnostizieren. Besonders deutlich wird dies sogar beim Frankfurter Hegel: Sprechen wir vom „Endlichen“ und „Unendlichen“, so bewegen wir uns in „Produkten bloßer Reflexion“. Als Reflexionsausdrücke verstellen diese Termini bereits, worum es geht. Sie schließen die Wirklichkeit nicht auf, sondern haben sie längst zerstört. Im Kontrast dazu setzt Hegel die Rede vom Leben ein. Leben ist kein „Produkt der Reflexion“, sondern konterkariert die Reflexion. Vom endlichen und unendlichen Leben zu sprechen, heißt nicht, das Endliche und Unendliche mit irgendeinem Zusatz zu versehen, als vielmehr die Sicht auf die Wirklichkeit vollständig zu verändern oder, besser gesagt, sie überhaupt erst zum Vorschein zu bringen.

Dass Jacobi diesen zentralen epistemischen Aspekt von Anfang an unterstreicht, ist nicht zu übersehen. „Von dem, was Leben ist, haben wir gewiß das innigste Bewustseyn; aber wer kann sich vom Leben eine Vorstellung machen?“ Vom Leben im Modus der Vorstellung zu sprechen, hieße, das Leben zum Objekt zu machen, mit der Folge, dass in dieser verobjektivierten Einstellung ein mir äußerlicher Gegenstand entstünde, der mit dem Leben gerade nichts mehr zu tun hätte. „Leben“ ist keine „Beschaffenheit“ der Dinge, es ist das Gegenteil dessen, worüber man prädikative Urteile fällen kann. Dass man dementsprechend einen Einstellungswechsel vollziehen und mitfolgend auch die Sprache verändern muss, um diesem Grundbefund gerecht zu werden und ihn überhaupt thematisch werden zu lassen, hat Jacobi bereits im Gespräch mit Lessing in die dann nicht zufällig prominent gewordene Formel gebracht, wonach es „das größeste Verdienst des Forschers“ ist, „Daseyn zu enthüllen und zu offenbaren“. ${ }^{13}$

Den Vorwurf des „Irrationalismus“, der gegen solche Intentionen von Seiten der Berliner Aufklärung erhoben und im 20. Jahrhundert durch Isaiah Berlin

13 Jacobi (1998), 29 (Hervorh. im Orig.). 
und Frederick Beiser noch einmal wiederholt und festgesetzt worden ist, sollten wir einfach als einen unsinnigen Vorwurf vergessen. Irrationalisten wären dann auch Schelling, Fichte und Hegel, die Jacobis Ausbruch aus den engen Rastern des Rationalitätsparadigmas folgen. Wörtlich übernimmt Schelling die Formel vom „Dasein enthüllen“, um einen „Gegenstand“ auszuzeichnen, der gerade kein begrifflich vermittelter Gegenstand, ,sondern das unmittelbare nur sich selbst Gegenwärtige im Menschen“ ist. Und auf diese Dimension bezieht sich auch Fichte, wenn er die Adresse an das Leben mit derjenigen an Erfahrung verbindet. Damit ist nicht das Erfahrungsmodell des Empirismus gemeint, das im Innenraum des Bewusstseins allenfalls die Verarbeitung eines Dateninputs vorsieht und noch nicht einmal die Referenz solcher Daten sicherstellen, geschweige denn die Wirklichkeit unseres In-der-Welt-Seins einholen kann. Das kann man zu Recht den „Mythos des Gegebenen“ nennen. Dass auch Erfahrung im kantischen Sinne die Sache nicht trifft, versteht sich nach allem ebenfalls von selbst. Die Alternative heißt Leben.

Unterdessen hat die Reflexion darauf, dass Leben kein irrationaler, aber sehr wohl ein präreflexiver (bzw. im weiteren Gang der hegelschen Philosophie ein ,spekulativer“) Sachverhalt ist, nicht nur den negativen Zweck, bestimmte Standarderwartungen rationaler Erklärung als sachinadäquat zurückzuweisen. Positiv gewendet kommt so das eigentliche Charakteristikum der lebensweltlichen Signatur des Lebensbegriffs zum Vorschein. Was Leben ist und was man im Namen des Lebens adressiert, ist in genau dem Maße kein Beobachtungsobjekt, wie es sich, mit Habermas gesagt, grundsätzlich nur aus der Teilnehmerperspektive verständlich machen lässt. Leben ist Ausdruck eines performativen Vollzugs, Ausdruck einer Praxis, die unabhängig davon, dass ich dabei und beteiligt bin, keine Bedeutung hat und umgekehrt alle Bedeutung aus solchem Vollzug gewinnt. Eine Disziplin, genannt „Praktische Philosophie“, ist dies selbstverständlich nicht. Mit dem performativen Sinn dieses Begriffs ist dann aber folgerichtig verbunden, dass er eine eigentümlich subjektive Prägung aufweist.

Wieder macht Jacobi hier den Anfang, wenn er von dem spricht, „was ich im eigentlichen Verstande mich selbst nenne“ - und in der Tat ist die Rede vom „Selbst“ den Begriffen des Subjekts oder des Ich jederzeit vorzuziehen. Wenigstens nominell können Subjekte auch Objekte sein; sofern sie dies wesentlich nicht sind, sind sie ein lebendiges Selbst. Ich komme auf diesen Punkt in meinem letzten Schritt zurück. An dieser Stelle will ich nur zweierlei unterstreichen. Nach allem bisher Gesagten ist erstens festzuhalten, dass das, was ich die eigentümlich subjektive Prägung des Lebensbegriffs genannt habe, weder „subjektivistisch“ auf irgendwelche privaten Dispositionen zielt noch auf die Subjektstellung der Bewusstseinsphilosophie, die ihre Stellung im Gegenzug gegen den mundanen Erfahrungsraum bezieht. Und zweitens scheint es mir völlig evident, dass die 
nachkantische Philosophie ihre charakteristische Hinsicht auf Subjektivität man erlaube für den Augenblick diese notdürftige Bezeichnung - dem nur performativ zu vergegenwärtigenden lebensweltlichen Interesse verdankt. In Schellings Text sticht dies förmlich hervor - es geht um das „lebendige Werk des menschlichen Geistes“ -, und zahllose Belege dafür ließen sich von Fichte anführen. Der Frankfurter Hegel nennt den praktischen Vollzug deshalb „Religion“ und ist zu dieser Zeit sogar der Meinung, dass Philosophie hier an ihre Grenze komme. Die „Erhebung des Menschen vom endlichen Leben zum unendlichen Leben“ ist ein Akt, keine Feststellung „positiv“ religiöser Lehren, und als Vollzug die Erfahrung derjenigen Wirklichkeit, die den Menschen im innersten betrifft.

Was ich damit sagen will: Es ist nicht Kants „transzendentale Apperzeption“, die die nachkantische Philosophie auf ihren Weg gebracht hat. Das scheint nur so zu sein - und wird darum auch immer von neuem bis heute zum Thema gemacht -, weil Fichte, Schelling und Hegel sich in immer neuen Anläufen an diesem „Ich denke“ abgearbeitet haben, nicht um diese Figur als unhintergehbaren Grund zu übernehmen oder auch nur daran anzuknüpfen, sondern um die „logische Funktion“ des Ich in ein lebendiges Selbst zu überführen.

\section{Individualismus oder Holismus: Lebensweltliche Differenzen}

Bevor ich zum nächsten Schritt übergehe, fasse ich den inzwischen erreichten Stand meiner Überlegungen kurz zusammen. Ich wollte zeigen, dass der Lebensbegriff in der klassischen deutschen Philosophie eine zentrale Rolle spielt. Dabei habe ich insbesondere für die These argumentiert, dass sich ausgehend von Jacobi auch bei Fichte, Schelling und Hegel das Interesse an diesem Begriff einem genuin lebensweltlichen Interesse verdankt. Mit diesem Interesse, so habe ich ferner argumentiert, sind eine Reihe von charakteristischen Merkmalen verbunden, die sich positionsübergreifend feststellen lassen und die vor allem keine randständigen Merkmale sind, sondern nichts Geringeres als das spezifische Format dieser Philosophie betreffen. Als ein im doppelten Sinne kritischer Begriff verweist der Einsatz des Metabegriffs „Leben“ auf den „realistischen“ Wirklichkeitsanspruch dieser Philosophie und in epistemischer Hinsicht zugleich auf ihren eigentümlichen rationalitätskritischen Zuschnitt. Dass das Leben kein Objekt ist, ist die Pointe dessen, um was es geht, und motiviert dazu, nach alternativen Ausdrucksweisen für ein Phänomen zu suchen, das sich wesentlich aus der Erfahrung der Teilnehmerperspektive, in der Praxis des Lebensvollzugs erschließt. Mit dieser Praxis der Selbst-Beteiligung hängt zugleich die Fokussierung auf „Subjektivität“ 
zusammen, die die Philosophie um 1800 charakterisiert und die sich in genau dem Maße von allen möglichen Theoriestellungen des Subjekts unterscheidet, wie es ein lebendiges Selbst ist, dem das Interesse gilt.

Es ist mir sehr bewusst, dass ich mit dieser Skizze ein Profil der klassischen deutschen Philosophie formuliere, das der Standardauffassung der Epoche „Von Kant bis Hegel“ in vielerlei Hinsicht widerspricht. ${ }^{14}$ Indessen habe ich bisher nur so etwas wie den allgemeinen Rahmen skizziert. Schon früher habe ich angedeutet, dass es Differenzen innerhalb der Positionen zu berücksichtigen gilt, und indem ich diese Differenzen jetzt thematisiere, kann ich zugleich konkretisieren, was mit der Adresse an das Leben aufgerufen wird, ohne dass es sich dabei - wie gerade noch einmal betont - um ein Objekt handelt, über das man aus der Beobachterperspektive Informationen gewinnen und Urteile fällen kann.

Einer der grundlegendsten Unterschiede zwischen Jacobi auf der einen und Fichte, Schelling und Hegel auf der anderen Seite besteht zweifelsohne darin, dass die drei zuletzt Genannten ein philosophisches Wissenschaftsprojekt betreiben, mit dem sie die Auffassung verbinden, dass Philosophie als System auftreten müsse. Diese Auffassung ist so eindringlich präsent, dass man sie unmöglich übersehen oder relativieren kann. Auf Anhieb scheint das nun im denkbar größten Kontrast zu meiner „lebensweltlichen“ Skizze zu stehen. Aus der Sicht Fichtes, Schellings und Hegels ist das jedoch nicht der Fall. Diejenige Wissenschaft, die ihnen vor Augen steht, soll ja gerade nicht den verfehlten Erkenntnisund Rationalitätsdiskurs der Moderne reproduzieren, sondern im Gegenteil dazu in der Lage sein, mit neuen epistemischen Mitteln der Wirklichkeit des Lebens zu entsprechen. Mit Hegel gesagt, geht es nicht um „Verstandesphilosophie“ als vielmehr um den Entwurf einer spekulativen Wissenschaft, die in der Folge dann auch konsequent zu einer neuen spekulativen Logik führt. Mit anderen Worten:

14 Die einzige mir bekannte Studie, die nicht zufällig ausgehend von Jacobi in eine ähnliche Richtung zielt, stammt von Horstmann (1991). Die neuere Darstellung von Förster (2011) bezieht Jacobi zwar an zentraler Stelle mit ein, ist jedoch im Ganzen rein erkenntnistheoretisch orientiert. Der Motivkomplex „System und Freiheit“ ist für die lebensweltliche Motivlage von zentraler Bedeutung: vgl. Jaeschke/Arndt (2012), 30-37. Ausdrücklich betone ich, dass ich mit dieser lebensweltlichen Rekonstruktion keine lebensphilosophische Deutung der klassischen deutschen Philosophie im Sinne der Strömung im späteren 19. und beginnenden 20. Jh. verfolge. Frontstellungen zwischen „Leben“ und „Geist“ oder zwischen „Leben“ und „Wissenschaft“ treffen die Konzepte Fichtes, Schellings und Hegels erkennbar nicht, und auch Jacobi, der ja durch Bollnow (1966) seinerzeit zu so etwas wie einem Ahnherrn der Lebensphilosophie gemacht werden sollte, ist dafür letztlich sogar in den Augen Bollnows selbst noch zu „rationalistisch“ eingestellt (was den Vorwurf des Irrationalismus auf bemerkenswerte Weise umkehrt). Eine nach wie vor hilfreiche Orientierung über Positionen der Lebensphilosophie im Ausgang von Schopenhauer bietet Schnädelbach (1983). 
Man muss die Sache im Auge haben, um die es der nachkantischen Philosophie geht, um ihre Bemühungen um ein Wissenschaftsprojekt zu verstehen, das übliche Erwartungen an szientifische Verfahren gezielt unterläuft.

Jacobi jedoch hat dieses Wissenschaftsprojekt dennoch nicht überzeugt, was im Fortgang der nachkantischen Philosophie bekanntlich für erhebliche Irritationen sorgt. Worin besteht der Kern dieser Interferenz? Wieso rechnet man mit Jacobis Zustimmung und ist konsterniert, als diese Zustimmung ausbleibt? Dafür muss es nach allem bisher Gesagten einen tieferen Grund geben, der die Ablehnung oder umgekehrt die Arbeit an einem philosophischen System affiziert. Dieser tiefere Grund verweist auf eine verschiedene Auffassung des Lebens selbst.

Was ich damit in Sicht bringen möchte, kann ich am besten am Fall Hegels zeigen, der im Jahr 1800 in Frankfurt ja noch gar kein Wissenschaftsprojekt verfolgt. Um so besser lässt sich die Differenz zu Jacobi bereits hier erkennen, gleichsam bereinigt um die Systemfrage, die in der Folge dann allerdings eine erhebliche Rolle spielt. Ich „müßte mich ausdrükken“, so Hegel in dem schon mehrfach erwähnten Fragment, „das Leben sey die Verbindung der Verbindung und der Nichtverbindung“" ${ }^{15}$ Das ist wohlgemerkt ein Ringen darum, in Ausdrücken der Reflexion zu formulieren, was eigentlich nur erfahren werden kann. Dafür steht der Verweis auf Religion. Wenngleich sich aber der Gehalt dieser Erfahrung im Reflexionsausdruck nur notdürftig abbildet, erhält man so doch einen wesentlichen Aufschluss: Leben ist erstens nichts Statisches, es ist eine Aktivität. Das reicht aber noch nicht zu, denn zweitens kommt es auf die Art dieser Aktivität an, die „Verbindung“ leistet. Damit setzt Leben drittens etwas Unverbundenes, die Mannigfaltigkeit von Vielem, voraus, auf das es sich zugleich bezieht. In diesem entscheidenden Sinn ist Leben nicht nur nichts Statisches, sondern vor allem auch keine vom Vielen abgespaltene Einheit. Leben ist eine komplexe Integration von Einheit und Vielheit, von „Identität und Differenz“, wie Hegel kurz darauf in der Differenzschrift formuliert.

Blickt man von hier aus zurück auf Jacobis Text, so stößt man zunächst einmal auf eine strukturell ähnliche Beschreibung. Leben ist keine Eigenschaft, die Dingen zukommt oder nicht. Umgekehrt, so Jacobi, drückt sich das Leben in den Dingen aus, ,denn das Mannichfaltige kann im Lebendigen allein sich durchdringen und Eins werden“. Etwas ist in genau dem Maße lebendig, wie es sich weder in Mannigfaltiges zerstreut noch sich jenseits davon als „mathematischer Punkt" fixiert. Lebendiges manifestiert sich als komplexe Integrationsleistung, als eine Einheit, die das Viele durchdringt und sich darin erhält. Diese Strukturform des Lebens nennt Jacobi mit Bezug auf Leibniz’ Monade die „substantielle

15 Hegel (2014), 343-344. 
Form“ eines Lebewesens, und ebenso spricht er in diesem Zusammenhang von der „Seele“, womit er evidentermaßen jeglichen Leib-Seele-Dualismus hinter sich lässt.

Wo liegt der nicht zu verkennenden strukturellen Verwandtschaft zum Trotz die Differenz zwischen Jacobi und Hegel? Zu beachten ist der letzte Satz in dem von mir zitierten Text Jacobis: „Wo aber Einheit, reale Individualität aufhört, da hört alles Daseyn auf.“ Der Fokus in Jacobis Lebens,,begriff“ liegt auf „realer Individualität“. Vom Leben zu sprechen, ohne im selben Moment zu implizieren, dass sich die Strukturform des Lebens im lebendigen Individuum manifestiert, ergibt keinen Sinn, und dies umso weniger, als es die Erfahrung unseres eigenen lebendigen Selbstseins ist, in der sich die Verfasstheit lebendiger Wirklichkeit ursprünglich erschließt. Eben diese Erfahrung legt Hegel hingegen seiner Beschreibung des Lebens nicht zugrunde. In seiner Darstellung bezeichnet das lebendige Individuum für sich genommen das Moment der Nichtverbindung, das Moment der Differenz, das nur dann kein Spaltprodukt der Reflexion ist, wenn es sich als Moment der übergreifenden Einheit des Lebens erfährt.

Mit anderen Worten: Was Hegel mit Reflexionsausdrücken zu sagen versucht und mit der „Erhebung vom endlichen Leben zum unendlichen Leben“ an die Religion adressiert, ist ein holistisches Verständnis des Lebens, das seine Ressourcen aus Spinozas „Hen kai pan“ bezieht. ${ }^{16}$ Gerade weil sich Hegel in seiner frühen Zeit mit Spinozas Philosophie nicht im entferntesten theoretisch befasst hat, liegt dieser Zusammenhang einer lebensweltlichen Aneignung des „Hen kai pan“ um so deutlicher zu Tage. Allein ist er damit allerdings nicht: „Was geht über die stille Wonne dieser Worte“, heißt es mit Bezug auf Spinoza in Schellings Ichschrift, „das Hen kai pan unsers bessern Lebens?“17

Die sämtlichen Umstände der ja wiederum von Jacobi beförderten „SpinozaRenaissance“ kann ich an dieser Stelle natürlich nicht einholen. Das ist auch nicht nötig, weil es mir hier allein darauf ankommt, auf den lebensweltlichen Hintergrund der Spinoza-Rezeption zu verweisen. Hier hat sich gleichsam eine „Weltanschauung“ kristallisiert, in der die Strukturform des Lebens als Totalität des Lebenszusammenhangs im Ganzen verstanden und Jacobis Insistenz auf dem Individuum daher zunächst auch ganz übersehen wird. Das gilt im übrigen nicht nur für Hegel und Schelling - Herder, Schleiermacher, die Romantiker und auch Fichte erleben und beschreiben die Welt ganz ähnlich. In gewisser Weise ist dies paradox: Aus der lebensweltlichen Teilnehmerperspektive wird die Sub-

16 Die für die Epoche ausschlaggebende Zuschreibung des „Hen kai pan“ auf Spinoza geht auf Lessing in Jacobis Darstellung von ihrer beider Gespräch zurück (Jacobi 1998, 16).

17 Schelling (1980), 111. 
sistenz des Individuums hier nicht gestärkt, sondern relativiert, aus derselben Perspektive des Beteiligtseins wird aber zugleich der Lebenszusammenhang im Ganzen „subjektiv“ interpretiert. In diesem Sinne sagt Hegel schon in Frankfurt, dass man „das unendliche Leben [...] einen Geist nennen“ kann. ${ }^{18}$

Wenn dem aber so ist, wenn es also in den Entwürfen der nachkantischen Philosophie einen solchen lebensweltlich holistischen „Subtext“ zu berücksichtigen gilt, dann folgt daraus nicht allein, dass man sich mit dem Lebensbegriff der Epoche nicht wie mit einer rein diskursiven Materie befassen kann. Es folgt vor allem auch, dass sich über die Interferenz der Ansätze nicht rein diskursiv entscheiden lässt. Ich gebe gerne $\mathrm{zu}$, dass es mir persönlich nicht gelingt, mich in den Erfahrungsraum eines lebensweltlichen Holismus hineinzuversetzen und die Vorzüge einer solchen Anschauung einzusehen. Aber es steht mir auch nicht zu, die Realität einer solchen Auffassung als unsinnig oder gar falsch zu bestreiten.

\section{Lebenswelt in systemischer Darstellung?}

Was sich allerdings diskursiv erörtern lässt, und mit dieser Überlegung komme ich zum Schluss, ist die Übersetzung des lebensweltlichen Holismus in die Form des Systems. Wiederum kann man diese Übersetzung am besten bei Hegel sehen, im Übergang von Frankfurt in die Abfassung der Differenzschrift, wo nicht von ungefähr die Totalität des Absoluten die Strukturform des Lebens übernimmt und sich die systemische Darstellung dieser Totalität der Wirklichkeit in Anlehnung an Schelling in die beiden Bereiche der Naturphilosophie und der Philosophie des Geistes zu differenzieren beginnt. ${ }^{19}$ Dass der naturphilosophische Lebensbegriff unter der Voraussetzung des lebensweltlichen „Metabegriffs“ steht, wie ich eingangs behauptet habe, wird damit belegt. Und mit Hegels an Schelling angelehnter These, dass auf beiden Seiten ein „Subjekt-Objekt“ anzunehmen sei, wird ferner auch belegt, dass das, was ich die eigentümliche Hinsicht auf „Subjektivität“ genannt habe, auch auf die Naturphilosophie selber durchschlägt. Den Gesamtzusammenhang der Natur als einen lebendigen Zusammenhang zu verstehen, heißt, die Natur nicht als Objekt, sondern als Subjekt zu begreifen, das sich evolutionär zum Bewusstsein hin entwickelt. ${ }^{20}$

18 Hegel (2014), 343.

19 Vgl. Sandkaulen (2017).

20 Wenn man den lebensweltlichen „Metabegriff“ in dieser Weise vom naturphilosophischen Begriff des Lebens unterscheidet, wird insbesondere auch klar, inwiefern Hegel unter dem Generalinteresse des Lebens das natürliche Leben einerseits und den Geist andererseits unterscheidet 
Ohne auf Schellings und Hegels Konzeptionen näher einzugehen, will ich hier nur ein Problem herausheben, das mir das Entscheidende zu sein scheint: Es besteht in der systematischen Explikation der Selbstbezüglichkeit, die eine lebendige Einheit sowohl vom toten Punkt als auch von der äußerlichen Zusammensetzung von zerstreutem Vielem unterscheidet. Das hier entstehende Problem lässt sich in beiden Richtungen verfolgen. Wenn gelten soll, dass das System über die Lebenswelt der Menschen nicht hinwegspricht, muss gezeigt werden können, dass ich genau dann bei mir selber bin, wenn ich mein Leben im Zusammenhang des Ganzen verstehe. Damit ist aber zugleich die Aufhebung eben derjenigen Identität verlangt, die mein individuelles Selbstsein ausmacht und die ich im Kontext mit anderen Individuen erfahre. Schelling hat diese Aporie als Aporie der „intellektuellen Anschauung“ sehr wohl gesehen und verschiedentlich umkreist.

Möchte man dieses Problem vernachlässigen, so stellt sich die Frage doch unvermeidlich in umgekehrter Richtung. Soll der Zusammenhang der Natur und der Welt des Geistes im Ganzen als ein selbstbezüglicher und eben deshalb lebendiger Zusammenhang systematisch dargestellt werden, so muss entweder die Identität als Prinzip des Systems fungieren, was zur Folge hat, dass das Prinzip den Charakter des lebendigen, auf eine Mannigfaltigkeit bezogenen Selbst verliert und zugleich unklar wird, wie eine solche Identität Differenz begründen und integrieren kann. Oder man privilegiert von Anfang an die Differenz und lädt sich damit dann die Schwierigkeit auf, das verbindende Moment in der Dynamik des Lebens aufzuweisen und zu zeigen, wie sich das Leben im einzelnen Lebewesen tatsächlich manifestiert.

Genau diese Probleme hat Hegel, das ist klar, mit dem Entwurf des ,spekulativen Begriffs“ zu lösen versucht. Allerdings halte ich diesen Begriff für ein Vexierbild. Man versteht, was Hegel meint, wenn man sich selbst an die Stelle dieses Begriffs setzt, aber in genau dem Maße, wie man dies tut, wird man von Hegel belehrt, dass die Subjektivität des Begriffs die Differenz der Individuen absorbiert. Nur in einem Fall ist dies nicht so, und mit dieser Pointe beende ich meine Ausführungen sehr gerne: Im Fall der hegelschen Anthropologie ist die Seele der „existirende Begriff“, sie ist ,in dem Leiblichen einfache allgegenwärtige Einheit“, die Hegel im Unterschied zur bloß natürlichen als „innerliche Individualität" bezeichnet. ${ }^{21}$ Es ist evident, dass Hegels anthropologische Darstellung nicht allein eine lebensweltliche (und keine naturphilosophische) Perspektive

und damit bei voller Anerkennung der Dimension der Natur einer „naturalistischen“ Lektüre seines Ansatzes enge Grenzen setzt. Aufschlussreich ist hier nicht zuletzt das Kapitel zum Selbstbewusstsein in der Phänomenologie des Geistes.

21 Hegel (1992), § 403 (Hervorh. im Orig.). Vgl. Sandkaulen (2010) sowie dies. (2012). 
geltend macht und aus dieser Perspektive des Lebens spricht. Es sieht auch ganz danach aus, dass er hier nun ohne Abstriche einschließlich der Adresse an die leibnizsche Monade den Intentionen Jacobis folgt. Die aktuelle Virulenz dieser Auseinandersetzung, die ich eingangs angekündigt habe, ergibt sich daraus. Denn die „life sciences“ muss man fragen, wie sie aus der Beobachterperspektive auf die „organisierten Einheiten“ feststellen wollen, wie es ist, ein lebendiges Selbst, eine „reale Individualität“ zu sein.

\section{Literatur}

Bollnow, O. F. (1966), Die Lebensphilosophie F. H. Jacobis [1933], Stuttgart.

Fichte, J. G. (1971), Sonnenklarer Bericht an das grössere Publicum, über das eigentliche Wesen der neuesten Philosophie. Ein Versuch, die Leser zum Verstehen zu zwingen, in: Fichtes Werke 2, hg. v. Fichte, I. H., Berlin, 323-420.

Förster, E. (2011), Die 25 Jahre der Philosophie. Eine systematische Rekonstruktion, Frankfurt am Main.

Habermas, J. (1981), Theorie des kommunikativen Handelns 2, Frankfurt am Main.

Hegel, G. W. F. (1968), Differenz des Fichteschen und Schellingschen Systems der Philosophie, in: Jenaer Kritische Schriften, Gesammelte Werke 4, hg. in Verbindung mit der Dt.

Forschungsgemeinschaft v. d. Nordrhein-Westfäl. Akad. d. Wiss. u. d. Künste, Hamburg, 5-92.

Hegel, G. W. F. (1992), Enzyklopädie der philosophischen Wissenschaften (= Gesammelte Werke 20), Hamburg.

Hegel, G. W. F. (1998), Introductio in Philosophiam, in: Schriften und Entwürfe (1799-1808) (= Gesammelte Werke 5), 259-265, Hamburg.

Hegel, G. W. F. (2014), Absolute Entgegensezung, in: Frühe Schriften II, hg. v. Jaeschke, W. (= Gesammelte Werke 2), 341-344, Hamburg.

Horstmann, R. P. (1991), Die Grenzen der Vernunft. Eine Untersuchung zu Zielen und Motiven des Deutschen Idealismus, Frankfurt am Main.

Jacobi, F. H. (1998), Über die Lehre des Spinoza in Briefen an den Herrn Moses Mendelssohn, in: Werke Gesamtausgabe 1,1, hg. v. Hammacher, K., u. Jaeschke, W., Hamburg, 1-146.

Jacobi, F. H. (2004), David Hume über den Glauben oder Idealismus und Realismus. Ein Gespräch, in: Werke Gesamtausgabe 2,1, Hamburg, 9-112.

Jaeschke, W. (2000), Zur Genealogie des Deutschen Idealismus. Konstitutionsgeschichtliche Bemerkungen in methodologischer Absicht, in: Arndt, A., u. Jaeschke, W. (Hg.), Materialismus und Spiritualismus. Philosophie und Wissenschaften nach 1848, Hamburg, 219-234.

Jaeschke, W., u. Arndt, A. (2012), Die Klassische Deutsche Philosophie nach Kant. Systeme der reinen Vernunft und ihre Kritik 1785-1845, München.

Sandkaulen, B. (2007), Jena als Chiffre des „deutschen Idealismus“. Motive und Folgen einer historischen Konstruktion, in: John, J., u. Ulbricht, J. H. (Hg.), Jena. Ein nationaler Erinnerungsort?, Köln u. a., 113-122. 
Sandkaulen, B. (2010), „Die Seele ist der existierende Begriff“. Herausforderungen philosophischer Anthropologie, in: Hegel-Studien 45, 35-50.

Sandkaulen, B. (2012), „Individuum est ineffabile“. Zum Problem der Konzeptualisierung von Individualität im Ausgang von Leibniz, in: Gräb, W., u. Charbonnier, L. (Hg.), Individualität. Genese und Konzeption einer Leitkategorie humaner Selbstdeutung, Berlin, 153-179.

Sandkaulen, B. (2017), Hegel's first System Program and the Task of Philosophy, in: Moyar, D. (Hg.), The Oxford Handbook of Hegel, New York, 3-30.

Sandkaulen, B. (2019), „Ich bin und es sind Dinge außer mir“. Jacobis Realismus und die Überwindung des Bewusstseinsparadigmas, in: dies., Jacobis Philosophie. Über den Widerspruch zwischen System und Freiheit, Hamburg, 135-167.

Sandkühler, H. J. (Hg.) (2005), Handbuch Deutscher Idealismus, Stuttgart u. Weimar.

Schelling, F. W. J. (1980), Vom Ich als Prinzip der Philosophie oder über das Unbedingte im menschlichen Wissen, in: Werke 2, Historisch-kritische Ausgabe, hg. v. Buchner, H., u. Jantzen, J., Stuttgart, 64-175.

Schnädelbach, H. (1983), Philosophie in Deutschland 1831-1933, Frankfurt am Main. 